\title{
Drogas e violência: percepção social em uma comunidade
}

\section{Drugs and violence: social perception in a community}

\section{Lúcia Margarete dos Reis ${ }^{1}$, Magda Lúcia Félix de Oliveira ${ }^{2}$}

\footnotetext{
${ }^{1}$ Enfermeira, Mestre em Enfermagem. Discente do Programa de Pós-Graduação em Enfermagem, nível Doutorado, da Universidade Estadual de Maringá (UEM). Coordenadora Centro de Atenção Psicossocial da Prefeitura Municipal de Sarandi. Sarandi, PR, Brasil. E-mail: luciamargarete@gmail.com.

${ }^{2}$ Enfermeira, Doutora em Saúde Coletiva. Professora Adjunto da UEM. Maringá, PR, Brasil. E-mail: mlfoliveira@uem.br.
}

\section{RESUMO}

Objetivou-se investigar a percepção social sobre drogas de abuso e violência em uma comunidade do Noroeste do Paraná. Pesquisa transversal, descritiva, utilizando questionário estruturado, aplicado a 358 moradores. Dos 358 entrevistados, 98,6\% referiram que percebem a presença de drogas em elevada intensidade $(82,4 \%)$, situação considerada "preocupante" para 56,1\% e motivo de sofrimento para 61,5\%. Setenta e oito entrevistados (22,1\%) informaram que a presença de drogas causou alteração em sua vida familiar $(22,1 \%)$ e social $(29,5 \%)$ e no comportamento familiar (24,9\%), e 72,6\% apontaram restrições em suas atividades pelo medo de sofrer violências. 0 principal motivo da circulação e consumo de drogas foi relacionado à ausência de policiamento (31,4\%). A maioria (90.2\%) percebe a presença de violência e destes $93,8 \%$ a relacionaram com o uso de drogas. A presença de violência foi majoritariamente relacionada ao uso de drogas, e ocorre devido à ausência de policiamento e combate ao tráfico na comunidade.

Descritores: Drogas Ilícitas; Violência; Percepção Social; Enfermagem Psiquiátrica; Enfermagem em Saúde Pública.

\section{ABSTRACT}

This cross-sectional, descriptive study aimed at investigating social perception on street drugs and violence in a community in northwestern Paraná. A structured questionnaire was applied to 358 inhabitants, of whom $98.6 \%$ reported to perceive the presence of drugs in high intensity (82.4\%), a situation considered as "alarming" for $56.1 \%$ and a cause of suffering for $61.5 \%$. Seventy-eight interviewees $(22.1 \%)$ reported that the presence of drugs caused changes in family life (22.1\%), social life (29.5\%), and in family behavior (24.9\%). A total of $72.6 \%$ reported restrictions in their activities due to fear of violence. The main reason for drug use and distribution was related to the absence of policing (31.4\%). Most people (90.2\%) perceived the presence of violence; $93.8 \%$ related this presence to drug abuse. The presence of violence was mostly related to drug abuse, as a result of the absence of policing and drug traffic fighting in the community.

Descriptors: Street Drugs; Violence; Social Perception; Psychiatric Nursing; Public Health Nursing. 


\section{INTRODUÇÃO}

O uso de drogas de abuso, consideradas como qualquer substância que ao ser introduzida no organismo provoca alterações no seu funcionamento, modificando uma ou mais funções, tem aumentado desde a década de 1990 e suas consequências na vida do indivíduo e da sociedade são consideradas um problema social e de saúde pública, no Brasil e no mundo. O Relatório Mundial sobre Drogas, publicado em 2011 revela que o número de usuários dessas substâncias passou de 180 milhões em 2009 para 210 milhões em $2010^{(1-2)}$.

O aumento do consumo de drogas e, consequentemente, da comercialização e ou tráfico, levou os moradores das comunidades à exposição das consequências desse contexto, marcado por problemas sociais, econômicos, legais, e de saúde, que envolvem violências e criminalidade, problemas no trabalho e desagregação de famílias. Algumas comunidades estão mais exposta às drogas de abuso, e ao impacto decorrente do seu uso, ampliando a percepção e a discussão sobre os problemas sociais existentes e o impacto das drogas na qualidade de vida e saúde da população ${ }^{(3-6)}$.

O aumento da violência ocorrido nas últimas duas décadas em Pesquisa realizada em municípios do Estado do Paraná identificou que o paranaense acredita que a violência está mais próxima de seu cotidiano. Sete em cada dez pessoas se sentem menos seguras hoje do que há cinco anos, e a sensação de perigo é respaldada nas estatísticas divulgadas pela mídia regional impressa que apontam, ano após ano, índices de criminalidade em tendência ascendente no Estado ${ }^{(7)}$.

As elevadas taxas de homicídio relacionadas ao uso e comercialização de drogas representam a ponta do iceberg da violência social. A percepção da violência é resultado da exposição frequente a imagens violentas nos meios de comunicação, e ao testemunho de atos violentos na própria comunidade, fortalecendo a crença que a violência é o desfecho natural e legítimo para muitos conflitos sociais ${ }^{(8)}$.
A população recebe informações sobre a violência relacionada à comercialização/tráfico e ao perigo do uso das drogas e, em contrapartida, é alvo de propagandas sofisticadas para estimular o consumo de bebida alcoólica e tabaco, que têm se destacado como precursoras do uso de drogas ilícitas ${ }^{(9)}$. Diante desta ambiguidade de informações, o reconhecimento da percepção social sobre o contexto que envolve o uso de drogas de abuso, e da influência que a violência exerce sobre a vida das pessoas nas comunidades, configura-se como importante ferramenta para a elaboração de estratégias de enfrentamento desse fenômeno ${ }^{(10-11)}$.

Desse modo, o presente estudo teve como objetivo investigar a percepção social sobre drogas de abuso e violência em uma comunidade.

\section{MATERIAL E MÉTODOS}

Estudo quantitativo, descritivo, e transversal, utilizando inquérito domiciliar de base populacional, realizado em um município da região Noroeste do Paraná.

O local da presente pesquisa foi um conjunto habitacional, inaugurado na década de 1990, destinado à famílias de baixa renda em que o responsável estivesse desempregado ou tivesse renda inferior ao salário mínimo vigente na época, e a família fosse constituída por cinco membros, com ou sem laços consanguíneos.

Para o delineamento do número de sujeitos foi considerada amostragem probabilística aleatória, representativa dos 5.140 moradores, estabelecendo nível de confiança de $95 \%$, erro amostral de 0.05 e valor de $p$ 0.10. A partir desse processo foi constituída amostra populacional de 358 pessoas, sendo entrevistado um morador, com idade igual ou superior a 18 anos, em cada domicílio. Um sistema de referência com visitas in loco à comunidade foi criado, utilizando um sistema com três estágios - sorteio das quadras, sorteio dos domicílios e sorteio do respondente presente no domicílio no momento da chegada da entrevistadora ao domicílio.

O questionário estruturado incluiu questões referentes ao perfil socioeconômico dos entrevistados, e 
à percepção sobre a presença de drogas na comunidade e as consequências na vida do entrevistado e da família. Esse questionário foi construído com embasamento no Sistema de Indicadores de Percepção Social (SIPS) do Instituto de Pesquisa Econômica Aplicada - IPEA ${ }^{(10)}$. O SIPS é uma pesquisa domiciliar realizada por meio de inquérito domiciliar junto às famílias brasileiras, com a finalidade de conhecer a percepção social da população sobre bens e serviços públicos, oferecidos em diversas áreas, e subsidiar análises e decisões sobre a formulação, implementação e avaliação de políticas públicas ${ }^{(10)}$.

A coleta de dados foi realizada nos meses de maio e junho de 2012. Os dados foram compilados em planilha eletrônica e o processamento e a análise dos dados foram realizados em software específico. A análise estatística consistiu em descrever os dados encontrados, por meio de análise descritiva simples (frequência absoluta, relativa, e cálculo das médias).

O projeto de pesquisa foi submetido à apreciação do Comitê de Ética e Pesquisa com Seres Humanos da Universidade Estadual de Maringá, recebendo parecer favorável ( $n$ o 6799/2012). Os indivíduos participaram da pesquisa somente após assinarem o Termo de Consentimento Livre e Esclarecido (TCLE).

\section{RESULTADOS}

\section{Caracterização dos sujeitos da pesquisa}

Os entrevistados possuíam idade média de 43,9 \pm 15 anos, a maioria era do sexo feminino (68,2\%) e escolaridade de grande parte deles era de nove a 11 anos de estudo, ou seja, até o Ensino Médio (36,3\%).

A renda familiar média dos entrevistados foi de $R \$ 1.602,00$, variando entre $R \$ 70,00$ e $R \$ 10.000,00$, considerando o salário mínimo vigente de $\mathrm{R} \$ 622,00$. Cento e sessenta e três $(45,5 \%)$ entrevistados referiram renda familiar inferior a dois salários $(R \$ 1.244,00)$. A maioria (53,6\%) referiu estar empregada ou desenvolvendo atividades autônomas como fonte de renda, embora o desemprego fosse referido por $12,9 \%$.
Das 244 mulheres entrevistadas, 29,9\% declararamse do lar, ou seja, trabalhavam exclusivamente para a própria família, não exercendo atividade remunerada fora do domicílio. As mulheres representaram 78,3\% dos 46 desempregados, pois 36 mulheres declararam a condição de desempregadas.

O tempo médio de residência na comunidade foi de $14,3 \pm 5$, variando de seis meses a 20 anos, sendo que a maioria dos moradores $89,1 \%$ vive em vizinhança permanente há mais de seis anos.

\section{Percepção social da presença de drogas de abuso e de violência na comunidade}

Dos 358 entrevistados, 98,6\% informaram que percebem a presença de drogas de abuso na comunidade em graus distintos de intensidade. Destes, a maioria respondeu que percebe a existência de drogas com intensidade elevada $81,3 \%, 13,1 \%$ referiram intensidade moderada, 2,8\% consideraram baixa intensidade e o desconhecimento da intensidade da presença de drogas e a percepção de ausência de drogas de abuso na comunidade foi referido por apenas 10 entrevistados $(2,8 \%)$.

Quando questionados sobre as alterações na vida familiar e social e as influências no cotidiano ocasionadas pela presença de drogas de abuso na comunidade, 56,1\% dos moradores entrevistados referiram que esta presença foi considerada "preocupante" e motivo de sofrimento para $61,5 \%$ (Tabela 1 ).

A presença de drogas de abuso na comunidade ocasionou interferência na vida familiar $(22,1 \%)$ e social $(29,5 \%)$ e no comportamento familiar $(24,9 \%)$ dos entrevistados (Tabela 1).

Embora a percepção da presença de drogas na comunidade tenha sido considerada como de baixa intensidade por 15 entrevistados (4,2\%), cinco deles referiram que a presença de drogas na comunidade é "preocupante" e oito moradores entrevistados referiram que esta presença causa sofrimento (Tabela 1). 
Tabela 1: Distribuição dos residentes entrevistados segundo a percepção sobre a presença de drogas na comunidade e as consequências na vida do entrevistado e da família. Maringá, PR, Brasil, 2012.

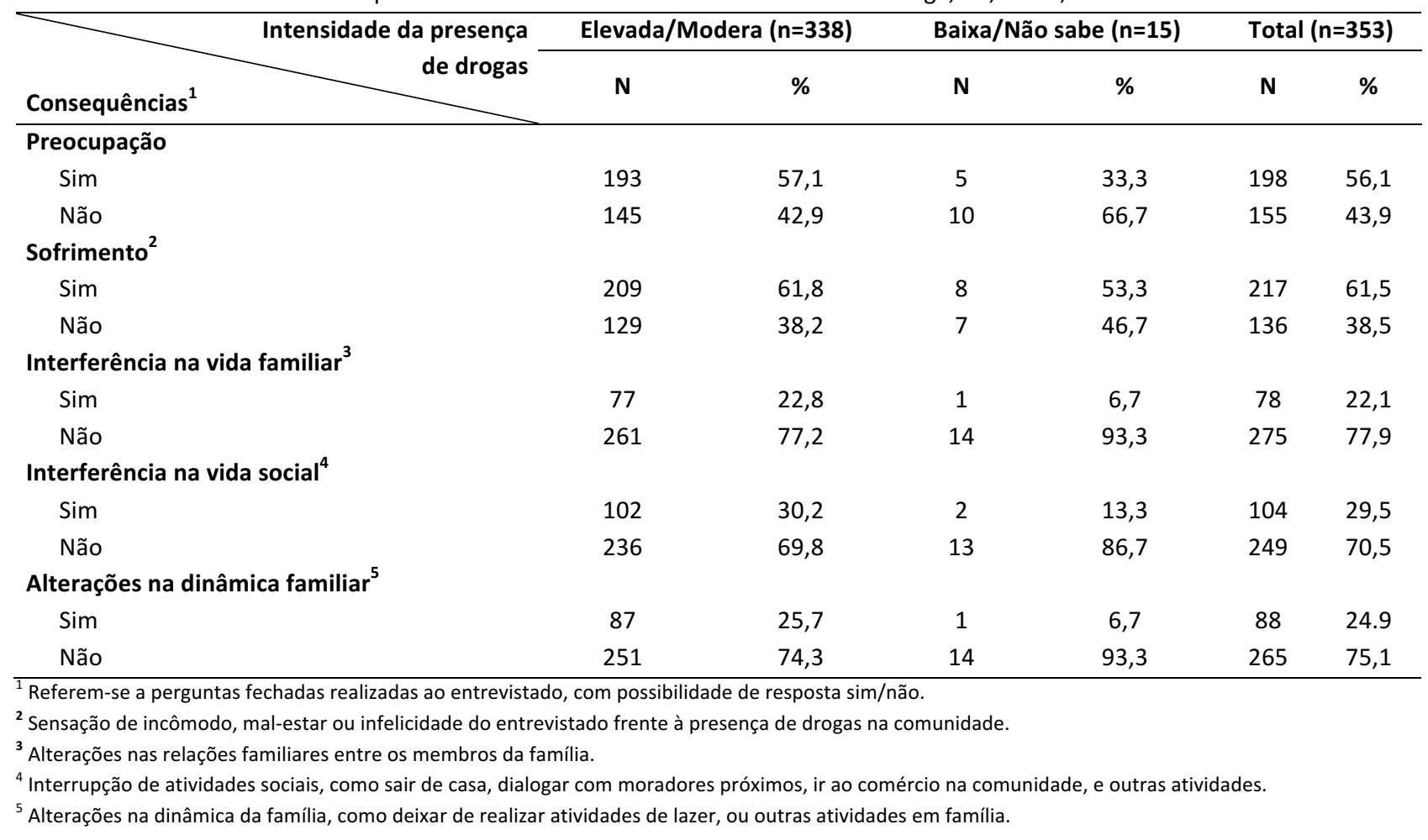

Ao serem questionados sobre o principal motivo da forte circulação e consumo de drogas de abuso na comunidade, $92,3 \%$ entrevistados elencaram oito motivos da existência das drogas de abuso na comunidade, que foram agrupadas em equipamentos de segurança pública, determinantes sociais, componentes familiares e componentes pessoais.

O principal motivo da circulação e consumo de drogas de abuso na comunidade foi relacionado aos equipamentos de segurança pública, representados pela ausência de policiamento e combate ao tráfico de drogas $(31,4 \%)$ e pela falta de punição dos usuários de drogas de abuso (2,5\%). Em relação aos componentes familiares, a desestrutura familiar foi motivo referido por $26,6 \%$ dos entrevistados. Os motivos relacionados aos determinantes sociais foram relacionados à ausência de educação, moradia e emprego (12,2\%), pobreza (1,7\%), ausência de acesso à cultura e lazer $(1,4 \%)$ e ausência de religião (0,9\%). E aos componentes pessoais, relacionouse a falta de conscientização da população para o não-uso $(15,6 \%)$.
Quanto à relação uso de drogas e violência, a maioria dos moradores $(90,2 \%)$ referiu que percebe a presença de violência na comunidade, destes, $93,8 \%$ a relacionaram com a presença do uso de drogas de abuso.

Dos 358 entrevistados, $8,7 \%$ sofreram atos violentos na comunidade, anterior à entrevista, sendo que as mulheres representaram $74,2 \%$ destes entrevistados. Dentre os tipos de violências referidos pelos entrevistados, a agressão física $(38,7 \%)$ e assalto $(35,5 \%)$ configuraram os tipos de violência frequentemente referidos pelos entrevistados, embora houvesse também agressão verbal $(19,4 \%)$ e tentativa de assassinato $(6,4 \%)$.

O medo de sofrer violências na comunidade foi referido por $79,6 \%$, dentre estas violências foram citadas assalto $(33,8 \%)$, bala perdida $(17,0 \%)$, assassinato $(15,9 \%)$, agressão física $(10,9 \%)$ e violência sexual $(2,0 \%)$.

Aos serem questionados sobre atividades cotidianas, $72,6 \%$ informaram que não realizam atividades como chegar ou sair de casa no período noturno $(58,5 \%)$, e sair de casa em qualquer hora do dia $(30,0 \%)$, frequentar festas e bares na comunidade (2,7\%) e dialogar com usuários de drogas $(8,8 \%)$. 


\section{DISCUSSÃO}

O perfil dos entrevistados corresponde a mulheres, adultos jovens, em idade economicamente ativa, e nível de escolaridade até o Ensino Médio, acompanhando as características sociodemográficas majoritárias na população brasileira ${ }^{(12-14)}$.

A renda familiar média dos entrevistados apresentou-se menor em relação ao rendimento médio mensal domiciliar da população brasileira, que correspondente atualmente a $\mathrm{R} \$ 2.419,00$. O percentual de entrevistados que, possuíam função remunerada, formal ou informal, se apresentou também inferior ao encontrado na população brasileira, que é de $54,2 \%$, e para as mulheres o percentual de entrevistadas que referiam desemprego (78,3\%) foi superior ao encontrado atualmente na população feminina brasileira, que corresponde a $59 \%^{(15)}$.

O tempo médio de residência na comunidade era de 14,3 anos, indicando vínculo do entrevistado com a vizinhança e os equipamentos sociais, o que poderia garantir maior fidedignidade às respostas. No entanto, a conivência por longo tempo com situações de violência pode levar à banalização desse problema na comunidade, pelo costume cotidiano.

Os resultados do presente apontam elevada percepção social da presença de drogas na comunidade, embora com graus distintos de intensidade, grande parte dos entrevistados referiu perceber a presença de drogas no cotidiano da comunidade com intensidade moderada a elevada, indicando a forte circulação e consumo de drogas de abuso na vizinhança.

Estudo realizado, a partir das fichas cadastrais de famílias utilizadas pelas equipes do Programa Saúde da Família na região onde se localiza a comunidade, já apontou o uso de drogas em seu cotidiano, sendo que o abuso de tabaco e de bebida alcoólica ocupava a terceira e a quarta posição, respectivamente, entre os agravos mais frequentes, confirmando a forte presença do uso de drogas de abuso na comunidade em estudo ${ }^{(16)}$.
Chama a atenção que a forte presença de drogas de abuso nesta comunidade influencia negativamente a vida da maioria dos moradores entrevistados, que se mostraram preocupados com a presença de drogas de abuso, sendo este um motivo desencadeador de sofrimento, segundo resposta dos entrevistados. Também, os resultados do estudo apontaram que entrevistados, mesmo considerando a presença de drogas de abuso na comunidade como de baixa intensidade, referiram a mesma influência das drogas em suas vidas.

A preocupação e o sofrimento com esta presença pode ser o resultado da convivência diária ou observação de pessoas fazendo uso de drogas e vivenciando os efeitos negativos desse uso. Vale ressaltar que, o conceito de sofrimento partiu do pressuposto mais subjetivo, cabendo ao entrevistado a resposta de acordo com a sua percepção deste termo. Sabe-se que a presença de drogas de abuso gera consequências devastadoras para o usuário, para a família e a comunidade onde este indivíduo está inserido, geralmente ocasionando alterações no comportamento familiar e nas comunidades $^{(3-4)}$.

Todavia, observou-se que os entrevistados, diante dessa situação considerada por eles como preocupante, em geral, não alteraram sua vida familiar, social ou o comportamento da família diante da presença do uso de drogas na comunidade. Embora, para aqueles que referiram influência das drogas de abuso em seu cotidiano, as alterações foram maiores quando relacionadas ao convívio social na comunidade.

Em relação ao motivo da forte circulação e consumo de drogas na comunidade, a falta de policiamento como equipamento de segurança pública foi o mais frequente entre os entrevistados. Também, o componente familiar e os determinantes sociais foram citados como motivadores da circulação e consumo de drogas.

A presença de drogas nas comunidades associa-se à ausência de políticas públicas para o combate ao uso de drogas e a fatores socioeconômicos desfavoráveis como 
condições inadequadas de moradia, baixa escolaridade, desemprego e pobreza, fatores também encontrados na comunidade em estudo ${ }^{(17)}$. O empobrecimento das famílias representa um risco adicional, pois a perda da capacidade de consumo pode levar jovens ao uso de drogas e à criminalidade, sendo o comércio de drogas de abuso, uma fonte de renda, em que a violência é a forma usualmente adotada para resolver conflitos e expandir a participação no mercado ${ }^{(18-19)}$.

$\mathrm{O}$ acesso às armas de fogo e a restrição das regras internas às "comunidades" facilita associação das atividades no tráfico de drogas a outros tipos de crime, como furtos, roubos e sequestros, o que amplia ainda mais a renda potencial de quem pratica o crime. 0 dinheiro obtido é garantia de usufruto imediato dos bens fundamentais à vida "social" do jovem da periferia: tênis sofisticados, telefones celulares de último tipo, roupas de marca e, se possível, motos e carros com acessórios brilhantes e aparelhagem de som ${ }^{(20)}$.

Quanto à relação ao uso de drogas e violência, os entrevistados referiram, também, forte presença de violência na comunidade. Este resultado corrobora a pesquisa realizada pelo Instituto Paraná Pesquisas no Estado do Paraná, que também encontrou a percepção de violência nas comunidades associada ao uso de drogas de abuso, em que $47 \%$ dos 1.505 entrevistados responderam que o uso de drogas de abuso é o fator mais importante relacionado à violência ${ }^{(7)}$.

No Brasil, a violência e as lesões traumáticas têm sido causas predominantes na morbimortalidade da população desde a década de 1980; até 2007, representavam $12,5 \%$ dos óbitos, especialmente entre os homens jovens (83,5\%). O padrão de ocorrência do trauma no Brasil não difere de outros países latinoamericanos, pois a maior parte dos óbitos é causada por homicídios ou está relacionada a acidentes de trânsito ${ }^{(21)}$.

A associação álcool e outras drogas e o aumento da violência urbana está associada como fator causal dos traumas por acidentes e por agressão, uma vez que, usuários de drogas de abuso apresentam comportamentos impulsivos e de exposição a riscos e quando combinados a um ambiente violento, potencializam a probabilidade de sofrer ou testemunhar eventos traumáticos ${ }^{(21)}$.

Quando questionados se foram vítimas de atos violentos na comunidade, $8,7 \%$ dos entrevistados responderam afirmativamente, e as mulheres representaram a maioria das vítimas de atos violentos nesta comunidade. Dentre os tipos de violências, a agressão física foi referida com maior frequência pelos entrevistados. No Brasil, o levantamento de dados do Sistema de Informações de Agravos de Notificação (SINAN), do Ministério da Saúde revela que as mulheres são as maiores vítimas de violência no país e entre os principais tipos de violência se destaca a agressão física, com 78,2\% dos casos de violência, dados semelhantes ao encontrado na presente comunidade ${ }^{(22)}$.

Segundo os resultados encontrados na comunidade em estudo, a presença de violência na comunidade gera sensação de insegurança e provoca medo nos indivíduos que convivem diariamente neste contexto. Em comunidades com forte pressão cultural para manter o uso e tráficos de drogas escondidos "debaixo do tapete", ou simplesmente para aceitá-los como "natural", os pequenos atos de violência possivelmente não serão totalmente relatados. As vítimas podem se recusar a discutir experiências violentas não somente por vergonha ou tabu, mas por medo ${ }^{(20)}$.

O medo de morrer foi frequentemente informado pelos entrevistados, expresso pelos tipos de violência relacionados à "bala perdida" e tentativa de assassinato. Os resultados do SIPS de 2012 também apontaram para essa tipologia de violência, indicando que $62,3 \%$ dos 2.888 entrevistados afirmaram ter muito medo de ser vítima de assalto à mão armada e tentativa de assassinato $(62,4 \%)^{(10)}$.

A população é frequentemente exposta aos impactos negativos da violência seja na comunidade ou através da mídia, esta que tem destacado a magnitude do impacto da violência para o indivíduo e a sociedade. As diversas 
formas de violência - assassinatos, tiroteios, sequestros, atos violentos contra crianças e jovens - ganham visibilidade e disseminação nos meios de comunicação. Esta exposição do indivíduo à violência na mídia pode suscitar a sensação de medo de ser vítima de atos violentos $^{(23)}$. Para os entrevistados o medo de assalto, medo de morrer por "bala perdida" e por tentativa de assassinato, pode ser reflexo da exposição a atos violentos na comunidade, mas por outro lado pode ser reflexo da exposição frequente à violência pelas informações fornecidas através dos meios de comunicação acessíveis à sociedade.

Mesmo dotado de um alto grau de subjetividade, o medo é um indicador que afeta a qualidade de vida da população, influenciado pela percepção do nível da ameaça de que tais eventos violentos realmente venham a ocorrer ${ }^{(10)}$. Dessa forma, o impacto da violência, principalmente para jovens, costuma ter efeitos negativos em seu desenvolvimento, e para adultos e idosos ocasionam mudanças de comportamento e alterações no estilo de viver ${ }^{(24)}$.

Os resultados do estudo apontaram também que os entrevistados ao serem questionados se sofreram alterações em sua vida familiar, social ou no comportamento da família, em decorrência da presença de drogas de abuso na comunidade, a maioria respondeu negativamente. No entanto, quando questionados sobre as atividades que evitam realizar por medo de sofrer violência na comunidade, a maioria respondeu que evita realizar algumas atividades na comunidade, destacandose sair do domicílio principalmente no período noturno e alguns entrevistados referiram que evitam sair a qualquer hora do dia. Tal situação indica que os moradores não alteraram suas vidas em decorrência do uso de drogas, mas em decorrência dos atos violentos inerentes a este uso.

Os resultados do estudo na comunidade corroboram os resultados encontrados em pesquisa realizada pelo Instituto Paraná Pesquisas, divulgada em mídia regional impressa. No Paraná, sete em cada dez pessoas se sentem menos seguras hoje do que há cinco anos, e a atividade que mais evitam fazer na comunidade por medo de sofrer violência é andar em ruas escuras, representada por $25 \%$ dos 1.505 entrevistados pelo Instituto Paraná Pesquisas $^{(7)}$.

Diante dessa informação, pode-se inferir que o consumo e a circulação de drogas de abuso estão presentes na comunidade em estudo e em outras comunidades do Estado. Também, a presença de drogas causa alterações no estilo de viver, porém a violência é o fator desencadeador de alterações no cotidiano dos entrevistados.

\section{CONCLUSÃO}

Os resultados apontam que a elevada presença de drogas na comunidade influencia negativamente a vida dos moradores, levando ao enclausuramento, pelo medo de sofrer atos violentos, com restrições ao trabalho para as mulheres, que deixaram de trabalhar para cuidar do domicilio, e diminuição de atividades cotidianas que exigem deslocamento dos entrevistados pelas ruas do bairro, principalmente no período noturno.

A ausência de policiamento e combate ao tráfico na comunidade foi considerado o motivo da forte circulação e consumo de drogas de abuso, e a presença de violência na comunidade foi majoritariamente relacionada ao uso de drogas.

Embora o estudo tenha sido realizado, em uma comunidade com elevada percepção do uso de drogas e violência, não se pode inferir que os resultados sejam representativos das demais comunidades do Estado do Paraná e do Brasil, uma vez que, a percepção social é influenciada pela cultura local e subjetividade dos indivíduos. No entanto, reforça a influência negativa do uso de drogas e da violência na vida das pessoas, e a necessidade de estudos que abordem as repercussões do uso de drogas nas famílias e na sociedade.

Também, faz-se necessário que os profissionais de saúde desenvolvam estratégias de prevenção do uso de drogas e empoderamento da população para conviver no 
contexto das drogas de abuso e da violência. Os profissionais de enfermagem são agentes-chave desse processo, pois possuem maior contato com a

\section{REFERÊNCIAS}

1. Jang HS, Kim JY, Choi SH, Yoon YH, Moon SW, Hong YS, et al. Comparative analysis of acutetoxic poisoning in 2003 and 2011: Analysis of 3 academic hospitals. J Korean Med Sci, 2013;28(10):1424-30.

2. United Nations. World drug report 2013. New York: United Nations Office on Drugs and Crime; United Nations; 2013. 151 p. Relatório final. [acesso em 05 mai 2015]. Disponível: http://www.unodc.org/unodc/secured/wdr/wdr2013/World_D rug_Report_2013.pdf.

3. Fertig A, Souza LM, Schneider JF. O cotidiano de familiares de usuários de crack: uma análise reflexiva. Rev enferm UFPE [Internet]. 2013 [acesso em: 25 mai 2015];7(esp):5726-32. Disponível em:

http://www.revista.ufpe.br/revistaenfermagem/index.php/revi sta/article/view/4837/pdf_3497

4. Marín-León L, Oliveira HB, Barros MBAo, Dalgalarrondo P, Botega NJ. Percepção dos problemas da comunidade: influência de fatores sócio-demográficos e de saúde mental. Cad. Saúde Pública, 2007; 23(5):1089-1097.

5. Otálvaro AFT, Álvarez GM, Velásquez TJD, Lugo ALH, Giraldo FLF, Suarez BAM et al . Calidad de vida de pacientes con dependencia a heroína de un centro de atención de drogodependencias de Medellín (Colombia). Invest Educ Enferm. 2012;30(1):35-46.

6. Seleghim MR, Galera SAF, Oliveira MLF. Pesquisa com Usuários de Crack e seus Familiares: Análise de uma de uma vivência. Sau. \& Transf. Soc. 2014;5(1):36-41.

7. Brembatti K. No PR, 70\% têm mais medo do que há 5 anos. Jornal Gazeta do Povo. 2011 maio 23; Paz tem voz: Caderno G. 8. Reis LM, Hungaro AA, Oliveira MLF. Políticas públicas para o enfrentamento do uso de drogas de abuso: percepção social em uma comunidade. Texto Contexto Enfermagem 2014;23(4): 1050-8.

9. Noto AR, Baptista MC, Faria ST, Nappo AS, Galduróz JCF, Carlini EA. Drogas e saúde na imprensa brasileira: uma análise de artigos publicados em jornais e revistas. Cad Saúde Pública, 2003; 19(1):69-79.

10. Instituto de Pesquisa Econômica Aplicada. Sistema de Indicadores de Percepção Social (SIPS) - Segurança Pública. Governo Federal. Secretaria de Assuntos Estratégicos da Presidência da República, 2012.

11. Marangoni SR, Oliveira MLF. Uso de crack por multípara em vulnerabilidade social: história de vida. Ciênc Cuid e Saúde. 2012;11(1):166-172.

12. Bernardy CCF, Oliveira MLF, Bellini LM. Jovens infratores e a convivência com drogas no ambiente familiar. Rev. RENE: Revista da Rede de Enfermagem do Nordeste. 2012;12(3):58996. comunidade, facilitando a identificação de problemas e a abordagem sobre o tema.
13. Laranjeira $\mathrm{R}$, organizador. II Levantamento nacional de álcool e drogas. São Paulo: Instituto Nacional de Ciência e Tecnologia para Políticas Públicas do Álcool e Outras Drogas; 2012. [acesso em: 27 mai 2015]. Disponível em: http://inpad.org.br/wp-content/uploads/2014/03/Lenad-IIRelat\%C3\%B3rio.pdf.

14. Instituto Brasileiro de Geografia e Estatística. Censo Demográfico 2010. [acesso em: 03 nov 2012]. Disponível em: http://www.ibge.gov.br.

15. Instituto Brasileiro de Geografia e Estatística. Pesquisa mensal de emprego, 2011. [acesso em: 03 nov 2012]. Disponível em:

http://www.ibge.gov.br/home/presidencia/noticias/noticia_vis ualiza.php?id_noticia=2222\&id_pagina $=1$.

16. Marcon SS, Nogueira LA, Fonseca ARO, Uchimura TT. Características da doença crônica em famílias residentes na região norte do município de Maringá, Estado do Paraná: uma primeira aproximação. Acta Scientiarum, 2004; 26(1):83-93. 17. Reis LM, Uchimura TT, Oliveira MLF. Socioeconomic and demographic profile in a vulnerable community to the use of drugs of abuse. Acta paul. enferm. [Internet]. 2013 [acesso em: 27 mai 2015];26(3):276-82. Disponível em:

http://www.scielo.br/scielo.php?pid=S0103210020130003000 $12 \&$ script $=$ sci_arttext\&tlng=en

18. Silva KL,Dias FLA,Vieira NFC,Pinheiro PNC. Reflexões acerca do abuso de drogas e da violência na adolescência. Esc Anna Nery [Internet]. 2010 [acesso em: 27 mai 2015];14(3):605-10. Disponível em: http://www.scielo.br/scielo.php?pid=S1414$81452010000300024 \&$ script=sci_arttext.

19. Malta DC, Mascarenhas MDM, Porto DL, Duarte EA, Sardinha LM, Barreto SM, et al. Prevalência do consumo de álcool e drogas entre adolescentes: análise dos dados da Pesquisa Nacional de Saúde Escolar. Rev Bras Epidemiol 2011; 14(1) Supl.:136-46.

20. Feltran GS. Sobre anjos e irmãos cinquenta anos de expressão política do "crime" numa tradição musical das periferias. Rev. Inst. Estud. Bras. 2013;56:43-72.

21. Imamura JH. Epidemiologia dos traumas em países desenvolvidos e em desenvolvimento [dissertação]. São Paulo: Universidade de São Paulo/USP; 2012. 144p.

22. Waiselfisz JJ. Mapa da Violência 2012 - Atualização: Homicídios de mulheres no Brasil. Rio de Janeiro: Centro Brasileiro de Estudos Latino-Americanos; 2012. [acesso em: 25 mai 2015]. Disponível em:

http://www.mapadaviolencia.org.br/pdf2012/MapaViolencia2 012_atual_mulheres.pdf

23. Zeitoune RCG, Ferreira VS, Silveira HS, Domingos AM, Maia AC. O conhecimento de adolescentes sobre drogas lícitas e ilícitas: uma contribuição para a enfermagem comunitária. Esc Anna Nery [Internt]. 2012 [acesso em: 25 mai 2015];16(1):57- 
63. Disponível em:

http://www.scielo.br/scielo.php?script=sci_arttext\&pid=S1414 $-81452012000100008$

24. Bernardy CCF, Oliveira MLF. O papel das relações familiares na iniciação ao uso de drogas de abuso por jovens institucionalizados. Rev. esc. enferm. USP [Internet]. 2010 [acesso em: 25 mai 2015];44(1):11-17. Disponível em:

http://www.scielo.br/scielo.php?script=sci_arttext\&pid=S0080 -62342010000100002 .

Recebido: 01/03/2014.

Aceito: $16 / 05 / 2014$.

Publicado: 30/09/2015. 